\title{
MODELOS DE AJUSTE E MÉTODOS PARA A DETERMINAÇÃO DA CURVA DE RETENÇÃO DE ÁGUA DE UM LATOSSOLO- VERMELHO-AMARELO ${ }^{(1)}$
}

\author{
Wanderley Andrade Costa ${ }^{(2)}$, Carlos Alberto da Silva Oliveira ${ }^{(3)} \&$ \\ Eiyti Kato ${ }^{(4)}$
}

\begin{abstract}
RESUMO
A caracterização da capacidade de retenção de água de um solo é fundamental para a descrição do fluxo de água através dele e para o adequado manejo da irrigação. São apresentadas comparações entre curvas de retenção de água do solo: ajustadas pelos modelos propostos por van Genuchten e por Hutson \& Cass; obtidas pelo método do WP4 usando processo de umedecimento e de secagem; obtidas pelo método da centrífuga utilizando amostras deformadas e indeformadas; e obtidas pelo WP4 e centrífuga, usando um processo de secagem e amostra deformada. Amostras deformadas e indeformadas foram coletadas com trados específicos em um Latossolo Vermelho-Amarelo (LVA), textura argilosa. Foram determinadas as propriedades hídricas do solo necessárias à elaboração das curvas de retenção de água obtidas por análise de regressão. $\mathrm{O}$ modelo de van Genuchten possibilitou o melhor ajuste nas diversas situações estudadas. Considerando o processo de secagem, o teor de água útil obtido superou em $13 \%$ o resultado do processo de umedecimento e evidenciou reduzido efeito de histerese. A amostra deformada apresentou-se com um teor de água útil superior $61,7 \%$ ao valor obtido para a amostra indeformada. Entre os métodos estudados, verificou-se que aquele que usa o WP4 subestimou os dados obtidos pela centrífuga. Verificou-se que há diferenças entre amostra deformada e indeformada e os métodos utilizados na obtenção da curva de retenção.
\end{abstract}

Termos de indexação: curva característica, van Genuchten, histerese, WP4.

\footnotetext{
(1) Recebido para publicação em agosto de 2006 e aprovado em outubro de 2007.

(2) Engenheiro-Agrônomo da Faculdade de Agronomia e Medicina Veterinária da Universidade de Brasília - FAV/UnB. Caixa Postal 4508, CEP 70910-970 Brasília (DF). E-mail: wander2601@yahoo.com.br.

(3) Professor Titular Doutor, FAV/UnB. E-mail: dasilvao@unb.br

${ }^{(4)}$ Pesquisador Associado, FAV/UnB. E-mail: kato@unb.br
} 


\title{
SUMMARY: ADJUSTMENT MODELS AND METHODS FOR DETERMINING THE SOIL WATER RETENTION CURVE OF A RED-YELLOW LATOSOL
}

\begin{abstract}
The characterization of the soil water retention capacity is fundamental for an adequate irrigation management and water flow description. We present comparisons of soil water retention curves adjusted in distinct ways: by the models proposed by van Genuchten and by Hutson \& Cass; by the WP4 method in a wetting and drying process; by the centrifuge method using disturbed as well as undisturbed soil samples; and by the WP4 and centrifuge using a drying process and disturbed soil samples. Disturbed and undisturbed samples were collected with a specific sampler in a Red-Yellow Latosol (Oxisol). Important soil water properties to establish the soil water retention curves, which were then determined by regression analysis. The van Genuchten model led to the best adjustment in the different studied situations. Based on the drying process, the available water content was $13 \%$ higher than by the wetting process, evidencing a reduced hysteresis effect. The available water content in the disturbed sample was $61.7 \%$ higher than in undisturbed samples. It was verified that among the studied methods the WP4 underestimated the data obtained by the centrifuge method. Differences were observed among procedures based on disturbed and undisturbed soil samples as well as the methods used to establish the retention curve.
\end{abstract}

Index terms: soil water characteristic curve, van Genuchten, hysteresis, WP4.

\section{INTRODUÇÃO}

A determinação da curva de retenção ou curva característica de água do solo é de suma importância na área de irrigação e nos estudos de movimento de água no solo, pois fornece o teor de água do solo $(\theta) \mathrm{em}$ diferentes tensões $(\Psi)$, e vice-versa. Assim, propicia condições necessárias para a determinação do teor de água disponível no solo, do teor de água atual e de outras variáveis básicos à execução do manejo adequado da água de irrigação e à quantificação dos processos dinâmicos envolvendo o sistema solo-plantaatmosfera.

Propriedades do solo, como teor de água de saturação $(\theta \mathrm{s})$ e teor de água residual $(\theta \mathrm{r})$, são necessárias e afetam a obtenção da curva de retenção de água do solo, $\theta(\Psi)$, e a curva de condutividade hidráulica não saturada, $K(\theta)$. Entretanto, na obtenção da curva de retenção são negligenciados importantes fatores ligados ao teor de água no solo, como histerese, difusão de vapor, expansão das argilas, temperatura, concentração de solutos na solução do solo, distribuição relativa do tamanho, da forma e do arranjo das partículas do solo (Hutson \& Cass, 1987; Moraes et al., 1993).

A curva de retenção de água pode ser obtida pelo processo de secagem da amostra previamente saturada ou por umedecimento gradual da amostra de terra fina seca ao ar (TFSA). As curvas obtidas por esses dois processos podem apresentar alguma diferença, devido ao fenômeno da histerese. Dentre outros aspectos, este fenômeno decorre da diferença do ângulo de contato da água que recua e avança no espaço poroso do solo, função do tipo de partículas minerais e orgânicas que favorecem a maior ou menor expansão ou contração dos colóides do solo, bem como da nãouniformidade dos raios dos poros capilares do solo. Para o manejo da irrigação, prefere-se o processo de secagem, entretanto, na simulação de processos de redistribuição da água no solo, não se deve negligenciar a histerese.

A histerese nas relações $\theta(\Psi)$ tem sido observada amplamente, para a maioria das amostras de solo, em laboratório (Iwata et al., 1988), não devendo ser negligenciada em campo. Este efeito é menor nas relações $\mathrm{K}(\theta)$. Entretanto há poucas informações sobre este fenômeno em Latossolos de cerrado.

A influência da temperatura na retenção de água do solo deve-se à maior ou menor intensidade das forças que retêm a água no solo a um dado potencial (Moraes et al., 1993). Segundo Klute (1986), esses efeitos da temperatura são ainda obscuros. Entretanto, assumindo um solo rígido, pode-se afirmar que a força de tensão superficial diminui com o aumento da temperatura e leva a um aumento no teor de água com base no fenômeno da capilaridade.

A textura e a estrutura do solo são importantes fatores na determinação das características dos poros, onde a água e o ar são retidos. Por exemplo, alguns solos argilosos de cerrado podem apresentar elevado índice de agregados, fazendo-os comportarem-se como solos arenosos, quanto à retenção de água (Adámoli et al., 1985). Santos (1997) relata que a porosidade total é maior em solos argilosos e menor em solos arenosos. Aqueles têm um número elevado de microporos, que contribuem para uma elevada capacidade de retenção de água e baixa permeabilidade, ao contrário dos arenosos. 
Inúmeros métodos de campo e de laboratório para a determinação da curva de retenção de água estão disponíveis na literatura. O que utiliza a câmara de pressão de Richards (Richards \& Fireman, 1943) possui a desvantagem de promover a paralisação da drenagem devido à interrupção do contato entre a amostra e a placa porosa, além da demora na obtenção dos pontos necessários para a elaboração da curva característica.

O método da centrífuga baseia-se na centrifugação de uma amostra de solo deformada e indeformada, previamente saturada. A cada rotação previamente escolhida está correlacionado um potencial matricial. Possui a vantagem da rapidez na obtenção da curva característica, além de envolver uma única saturação, minimizando os efeitos da histerese (Silva, 1980). Como desvantagem, há a presença de grumos, que dificultam e podem limitar o fatiamento e a compactação da amostra (Freitas Junior \& Silva, 1984).

O método denominado WP4 (Dewpoint Potentia Meter) para obtenção da curva característica consiste em um aparelho que realiza leituras rápidas, em torno de 5 min, do potencial de água de uma amostra deformada do solo, a partir do equilíbrio entre a fase vapor da água e a fase líquida presente na amostra dentro da câmara de leitura do aparelho, onde esta fica hermeticamente fechada, medindo então a pressão de vapor do ar. A partir da equação geral de estado $(\mathrm{PV}=\mathrm{nRT})$, a relação entre o potencial da água da amostra ( $\Psi)$, em $\mathrm{MPa}$, e a umidade relativa do ar do solo $\left(\mathrm{e} / \mathrm{e}_{\mathrm{o}}\right)$ é dada pela equação:

$$
\Psi=(\mathrm{R} \mathrm{T} / \mathrm{M}) \ln \left(\mathrm{e} / \mathrm{e}_{0}\right)
$$

em que e é a pressão do vapor do ar do solo, $\mathrm{e}_{0}$ é a pressão de saturação de vapor do ar na mesma temperatura do ar da amostra (e e e e em $\mathrm{kPa}$ ), R é a constante de gás $\left(8,31 \mathrm{~J} \mathrm{~mol}^{-1} \mathrm{~K}^{-1}\right)$, Té a temperatura em Kelvin da amostra e M é a massa molecular da água. A pressão do vapor do ar pode ser medida usando um espelho resfriado e $e_{0}$ é dado em função da temperatura da amostra.

A pressão do vapor do ar na câmara é considerada como a pressão do vapor do ar saturado na temperatura do ponto de orvalho. Quando os potenciais de água da amostra e do ar na câmara estão em equilíbrio, a medida da pressão de vapor na câmara e a temperatura da amostra possibilitam o cálculo do potencial de água da amostra (Biophysical..., 2004). Possui a vantagem de proporcionar rápidas leituras, e a desvantagem de ser afetado por mudanças bruscas de temperatura do ambiente e apresentar baixa precisão em potenciais da água próximos e abaixo de 0,1 MPa.

Diversas expressões para $\theta(\mathrm{h})$ existem na literatura. Leij et al. (1997) explicitam que as expressões utilizadas para quantificar as propriedades hidráulicas do solo são comumente usadas em programas de computador para modelar o fluxo e o transporte subsuperficiais em meios porosos e investigar modos indiretos para estimar estas propriedades. Estes autores estudaram diferentes funções utilizadas para descrever as relações $\Psi(\theta)$ em solos não saturados e as que proporcionaram maiores valores de $\mathrm{R}^{2}$ foram as propostas por van Genuchten (1980) e Hutson \& Cass (1987). Estas equações são dependentes de apenas dois parâmetros de ajuste estatístico e serão tratadas a seguir.

Van Genuchten (1980) propôs o seguinte modelo para a descrição matemática da relação funcional entre o teor e a tensão de água do solo:

$$
\begin{gathered}
\operatorname{Se}=\left[1+(\alpha h)^{n}\right]^{-m} \\
\theta=\left\{(\theta \mathrm{S}-\theta \mathrm{r}) /\left[1+(\alpha \mathrm{h})^{n}\right]^{m}\right\}+\theta \mathrm{r}
\end{gathered}
$$

em que $\theta$ é o teor de água do solo, $\theta$ r é teor de água residual medido ou ajustado por regressão; $\theta$ s é o teor de água de saturação do solo, todos com unidade em $\mathrm{cm}^{3} \mathrm{~cm}^{-3}$; "Se" é a saturação efetiva dada pelo quociente $(\theta-\theta \mathrm{r}) /(\theta \mathrm{s}-\theta \mathrm{r})$; $\alpha$ é um parâmetro de ajuste, em $\mathrm{cm}^{-1} ; \mathrm{h}$ é a tensão de água no solo em $\mathrm{cm}$; e m e $\mathrm{n}$ são parâmetros de ajuste do modelo.

Os parâmetros empíricos m e $\mathrm{n}$ podem ser estimados mediante análise de regressão não linear, pelo método dos quadrados mínimos, utilizando recursos computacionais (van Genuchten, 1980; Dourado Neto et al., 1990).

Para evitar problemas decorrentes de descontinuidade da função exponencial $\Psi(\theta)$ para valores próximos ao potencial de entrada de ar ou tensão de borbulhamento, Hutson \& Cass (1987) propuseram um modelo proveniente das funções estabelecidas inicialmente por Brooks \& Corey (1966) juntamente com o proposto por Campbell (1974) em que o teor de água residual é ignorado $(\theta r=0)$. Segundo Hutson \& Cass (1987), a representação da curva característica é obtita em duas partes: uma primeira para tensões maiores que a do ponto de inflexão, e uma segunda para tensões menores ou iguais à tensão do ponto de inflexão, que resultou no seguinte modelo, relacionando teor de água $\theta \mathrm{em} \mathrm{cm}^{3} \mathrm{~cm}^{-3}$ e tensão $\mathrm{h} \mathrm{em} \mathrm{cm}$ :

$$
\begin{gathered}
\operatorname{Se}=(\alpha \mathrm{h})^{-\beta} \\
\theta=(\theta \mathrm{s}-\theta \mathrm{r})(\alpha \mathrm{h})^{-\beta}+\theta \mathrm{r} \quad \mathrm{h}>\mathrm{hi} \\
\mathrm{Se}=1-(\alpha \mathrm{h})^{2}\left(1-\mathrm{Si}^{2}\right) \mathrm{Si}^{2 b} \\
\theta=\theta \mathrm{s}\left[1-(\alpha \mathrm{h})^{2}(1-\mathrm{Si}) \mathrm{Si}^{2 b}\right] \quad \mathrm{h} \leq \mathrm{hi}
\end{gathered}
$$

em que $\alpha$ é identificado como o recíproco do valor de entrada de ar no solo $\left(\mathrm{cm}^{-1}\right)$; h é a tensão de água no solo $(\mathrm{cm}) ; \beta$ é um parâmetro empírico obtido da equação resultante da análise de regressão, que relaciona "Se" com a tensão h; "Si" é o parâmetro resultante da relação entre teor de água no ponto de inflexão e teor de saturação $(\theta \mathrm{i} / \theta \mathrm{s})$; b é parâmetro equivalente a $1 / \beta$; e $\theta \mathrm{i}$ é dado pela relação $(2 \mathrm{~b} \theta \mathrm{s}) /$ $(1+2 b)$. Por conseguinte, a tensão no ponto de inflexão (hi) correspondente ao $\theta \mathrm{i}$, foi calculada por: $\mathrm{hi}=-\mathrm{a}$ $[(2 \mathrm{~b}) /(1+2 \mathrm{~b})]^{-\mathrm{b}}$, em que "a" é um coeficiente obtido pela relação $\mathrm{a}=\left(\alpha^{\beta}\right)^{1 / \beta}$, proveniente da análise de regressão. 
O objetivo deste trabalho foi comparar as curvas de retenção de água do solo ajustadas pelos modelos propostos por van Genuchten e por Hutson \& Cass; obtidas por um processo de umedecimento e de secagem, utilizando o método do WP4; as obtidas pelo método da centrífuga, utilizando amostras deformadas e indeformadas; e obtidas pelo WP4 e centrífuga, usando um processo de secagem e amostra deformada.

\section{MATERIAL E MÉTODOS}

Este trabalho utilizou dados de tensão e teor de água de amostras deformadas obtidos pelo método WP4 (Dewpoint PotentiaMeter), por meio dos processos de secagem e umedecimento, e dados de amostras deformadas, obtidos por Santana (2003), usando o método da centrífuga e também com amostras indeformadas.

As amostras de solo foram coletadas em um Latossolo cultivado com cafeeiro, implantado na Fazenda Água Limpa - Universidade de Brasília, Distrito Federal, e apresentaram as seguintes características: Latossolo Vermelho-Amarelo (LVA) (Embrapa, 1999); textura argilosa; teor de matéria orgânica de 3,8 dag kg-1; $\mathrm{pH}$ de 5,$3 ; \mathrm{Al}^{3+}=0,6 \mathrm{mmol}_{\mathrm{c}} \mathrm{dm}^{-3}$; $\mathrm{Ca}+\mathrm{Mg}=18,4 \mathrm{mmol}_{\mathrm{c}} \mathrm{dm}^{-3} \mathrm{e} \mathrm{H}+\mathrm{Al}=69,4 \mathrm{mmol}_{\mathrm{c}} \mathrm{dm}^{-3}$. As coletas de amostra deformada e indeformada foram feitas com a ajuda de trados holandês e uhland, respectivamente, em pontos aleatórios, na profundidade de $0-20 \mathrm{~cm}$.

A amostra homogeneizada passou por uma peneira de $2 \mathrm{~mm}$, sendo posteriormente seca ao ar durante uma semana (TFSA). Esta TFSA serviu para A determinação da relação $\psi(\theta)$ por processos de umedecimento e secagem em amostras deformadas.

A densidade do solo foi obtida usando anéis volumétricos com volume conhecido, tanto para amostras deformadas com TFSA quanto para indeformadas. As amostras deformadas e indeformadas foram secas pelo método da estufa. Após esse processo, pesou-se novamente, obtendo-se a massa de sólidos. A densidade média do solo foi obtida pela relação entre massa de sólidos e volume, com quatro repetições.

O teor de água residual foi obtido em laboratório e campo com quatro repetições e calculado pela relação entre massa de solo seco ao ar menos massa de sólidos sobre massa de sólidos (Embrapa, 1997).

Método WP4, por umedecimento - A curva de retenção por umedecimento da amostra foi obtida de 12 subamostras de TFSA, onde em cada uma foram adicionadas diferentes massas de água destilada nas seguintes proporções em $\mathrm{g}$ de água por g de solo: 0,15 ; 0,$175 ; 0,20 ; 0,21 ; 0,22 ; 0,23 ; 0,24 ; 0,25 ; 0,26 ; 0,27$; 0,28 ; e 0,29 .

De cada subamostra, foram retirados de 2 a $4 \mathrm{~g}$, e colocadas no recipiente de amostragem do WP4 (dimensões: $40 \mathrm{~mm}$ de diâmetro e 11,5 mm de altura), de tal forma que o nível de solo ficasse abaixo da metade do recipiente de amostragem, evitando sujar o bloco sensor e causar erro de leitura, conforme manual de operações do equipamento.

Em cada subamostra, foram realizadas quatro determinações para estimar a média do potencial de água do solo. Em cada determinação, foi considerada a média de duas leituras consecutivas do WP4. Após as leituras, cada amostra foi pesada e colocada em estufa por $24 \mathrm{~h}$ para o cálculo do teor de água $(\theta \mathrm{m})$ da amostra, pela relação entre a massa de água (ma) e a massa de sólidos (mp).

O teor de água de saturação $(\theta \mathrm{s})$ foi obtido saturando a amostra com o auxílio de uma bureta até que a superfície desta tornou-se visualmente brilhosa. Foi calculado pela relação $\theta \mathrm{s}=(\mathrm{ma} / \mathrm{mp}) \rho \mathrm{s}$, sendo $\rho \mathrm{s}$ a densidade do solo em $\mathrm{g} \mathrm{cm}^{-3}$. A massa de água da amostra foi determinada pela soma da massa de água adicionada pela bureta mais a massa de água residual da amostra. A massa de sólidos foi determinada pelo método da estufa.

Método WP4, por secagem - Na obtenção da curva de retenção pelo processo de secagem também foram utilizadas 12 subamostras de TFSA. A cada subamostra de 25,9g foram adicionadas $17,6 \mathrm{~g}$ de água destilada, de forma que esta fosse preenchendo os poros por capilaridade, tornando-as saturadas. As massas de TFSA e de água foram definidas em função do tamanho do recipiente utilizado e do teor de água do solo saturado de $0,605 \mathrm{~cm}^{3} \mathrm{~cm}^{-3}$. Posteriormente, cada subamostra saturada foi pesada e mantida em estufa entre $105-110{ }^{\circ} \mathrm{C}$ por intervalos de tempos diferentes, variando de 30 a $50 \mathrm{~min}$, ajustados pelos autores, de modo a se chegar a teores de água decrescentes, mas próximos daqueles utilizados no processo de umedecimento.

Após ter sido retirada da estufa, foram coletados subamostras em média de 2 a 4 g de solo e colocados no recipiente de amostragem do WP4. A leitura do potencial de água pelo WP4 foi feita de modo análogo ao processo de secagem.

A faixa de variação da temperatura da amostra usando o WP4 para a obtenção da curva característica em um processo de umedecimento foi de 25,2 a $28,8^{\circ} \mathrm{C}$ e pelo processo de secagem foi de 22,5 a $27,7^{\circ} \mathrm{C}$, sem variações consideráveis entre as faixas de temperatura em ambos os processos.

Método da centrífuga, amostra deformada e indeformada - $\mathrm{O}$ método da centrífuga utilizado para a obtenção da curva de retenção nas tensões de 0 ; $6 ; 10 ; 33 ; 60 ; 100 ; 500 ;$ e $1.500 \mathrm{kPa}$ foi o mesmo descrito por Santos (1997). Foram utilizadas amostras de solo deformadas e indeformadas com duas repetições para cada tensão avaliada. As análises foram realizadas no laboratório da Embrapa Cerrados. As amostras foram saturadas por capilaridade, sendo posteriormente centrífugadas por $30 \mathrm{~min}$, em determinada rotação, 
correspondente a uma tensão aplicada à amostra (Freitas Júnior \& Silva, 1984). O teor de água no solo, em cada tensão, foi obtido pelo método da estufa.

Os dados da curva de retenção de água usando o WP4 (processo de secagem e umedecimento) e os dados da centrífuga (amostra indeformada e deformada) foram ajustados pelos modelos de Hutson \& Cass (1987) e de van Genuchten (1980) em que os parâmetros empíricos $\mathrm{m}$ e $\mathrm{n}$ foram estimados assumindo relação de dependência entre si, segundo Mualem (1976).

O modelo de Hutson \& Cass foi ajustado com o auxílio do aplicativo Excel da Microsoft, e o modelo de van Genuchten com o auxílio do aplicativo Soil Water Retention Curves (SWRC version 3.00 beta), desenvolvido por Dourado-Neto et al. (2001).

Comparações entre os dois modelos usados e processos de estabelecimento das curvas características foram feitas com base no valor do coeficiente de determinação $\left(\mathrm{R}^{2}\right)$ e nas comparações entre os parâmetros das equações de regressão linear ajustadas entre valores de interesse e uma linha 1:1. Complementando a análise dos dois modelos, foi utilizado o método proposto por Camargo \& Sentelhas (1997), sendo a determinação de um índice de confiança "c", que analisa o desempenho de ajustes, feita pelo produto $c=R d$, sendo $R$ o coeficiente de correlação e "d" o índice de Willmont, que é obtido por:

$$
\mathrm{d}=1-\left\{\sum_{\mathrm{i}=1}^{\mathrm{n}}\left(\hat{\mathrm{e}}_{\mathrm{i}}-\mathrm{e}_{\mathrm{i}}\right)^{2} / \sum_{\mathrm{i}=1}^{\mathrm{n}}\left(\left|\hat{\mathrm{e}}^{\prime} \mathrm{i}\right|+\left|\mathrm{e}^{\prime} \mathrm{i}\right|\right)^{2}\right\}
$$

em que d é um índice que varia de 0 a $1 ; \hat{e}_{i}$ é o teor de água no solo, estimado pelo modelo; $\mathrm{e}_{\mathrm{i}}$ é o teor de água no solo observado, ambos em $\mathrm{cm}^{3} \mathrm{~cm}^{-3}$. Os valores de $\hat{e}_{i}^{\prime}$ e e ${ }_{i}$ são obtidos pelas relações: $\hat{e}_{i}^{\prime}=\hat{e}_{i}-\bar{e}$ e e ${ }_{i}=e_{i}-$ $\overline{\mathrm{e}}$, onde è é a média dos valores observados. O desempenho do ajuste foi obtido com o enquadramento do valor do índice de confiança "c" nas faixas: $>0,90$, ótimo; entre 0,81 e 0,90, muito bom; entre 0,71 e 0,80, bom; entre 0,51 e 0,70, mediano; entre 0,41 e 0,50, sofrível; entre 0,31 e 0,40, mau; e $\leq$ 0,30, péssimo.

A água disponívell foi obtida pela diferença entre os teores de água equivalente as tensões de 15.000 e $100 \mathrm{~cm}$.

\section{RESULTADOS E DISCUSSÃO}

Pelos dados do quadro 1, os valores de $\alpha$ variaram entre 0,2075 e 7,67 segundo o procedimento utilizado. Aqueles obtidos pelo modelo de Hutson \& Cass foram superiores aos de van Genuchten, exceto no processo de umedecimento. Os valores desta variável obtidos por Carsel \& Parrish (1988) variaram com a textura do solo, sendo de 0,008 em um solo argiloso e de 0,145 em um solo arenoso. Entretanto, os valores encontrados neste trabalho usando solo argiloso foram mais elevados, e podem ter sido afetados pela agregação da argila presente neste Latossolo.

Dentro de cada método e processo analisado, o modelo de ajuste proposto por van Genuchten proporcionou maiores valores de $\mathrm{R}^{2}$ em comparação com o modelo de Hutson \& Cass. A maior diferença no valor de $\mathrm{R}^{2}$ foi obtida dentro do método da centrífuga com amostra deformada (Quadro 1).

Quadro 1. Valores dos parâmetros de ajuste $(\alpha, \mathrm{b}, \mathrm{m}, \mathrm{n}$ ou $\beta)$, variáveis independentes $(\theta \mathrm{s}, \theta \mathrm{r}$ e $\mathrm{Si}=\theta \mathrm{i} / \theta \mathrm{s}) \mathrm{e}$ coeficiente de determinação $\left(R^{2}\right)$ dos modelos de van Genuchten (vG) e de Hutson \& Cass (HC) para os dados da curva de retenção obtidos pelos métodos da centrífuga e WP4, em um Latossolo VermelhoAmarelo (LVA) de cerrado, Brasília/DF

\begin{tabular}{|c|c|c|c|c|c|c|c|c|}
\hline \multirow{3}{*}{ Parâmetro } & \multicolumn{8}{|c|}{ Método } \\
\hline & \multicolumn{2}{|c|}{$\begin{array}{l}\text { Centrífuga } \\
\text { (deformada) }\end{array}$} & \multicolumn{2}{|c|}{$\begin{array}{c}\text { Centrífuga } \\
\text { (indeformada) }\end{array}$} & \multirow{2}{*}{$\begin{array}{l}\text { WP4 } \\
\text { vG }\end{array}$} & \multirow{2}{*}{$\begin{array}{c}\text { Secagem } \\
\text { HC }\end{array}$} & \multicolumn{2}{|c|}{$\begin{array}{c}\text { WP4 } \\
\text { umedecimento }\end{array}$} \\
\hline & vG & $\mathrm{HC}$ & $\mathrm{vG}$ & $\mathrm{HC}$ & & & vG & $\mathrm{HC}$ \\
\hline$\alpha\left(\mathrm{cm}^{-1}\right)$ & 0,2075 & 0,529 & 0,6767 & 0,704 & 2,6754 & 4,292 & 7,6777 & 3,690 \\
\hline $\mathrm{m}^{(1)}$ & 0,1388 & - & 0,1161 & - & 0,1100 & - & 0,1010 & - \\
\hline $\mathrm{n}$ & 1,1611 & & 1,1314 & & 1,1236 & & 1,1124 & \\
\hline$\beta$ & & 0,123 & & 0,095 & & 0,102 & & 0,104 \\
\hline$b^{(2)}$ & - & 8,130 & - & 10,493 & - & 9,833 & - & 9,625 \\
\hline $\mathrm{Si}$ & - & 0,942 & - & 0,955 & - & 0,952 & - & 0,951 \\
\hline$\theta \mathrm{i}\left(\mathrm{cm}^{3} \mathrm{~cm}^{-3}\right)$ & 0,703 & 0,663 & 0,587 & 0,563 & 0,527 & 0,576 & 0,484 & 0,575 \\
\hline$\theta \mathrm{s}^{(3)}\left(\mathrm{cm}^{3} \mathrm{~cm}^{-3}\right)$ & 0,704 & 0,704 & 0,590 & 0,590 & 0,605 & 0,605 & 0,605 & 0,605 \\
\hline$\theta \mathrm{r}^{(3)}\left(\mathrm{cm}^{3} \mathrm{~cm}^{-3}\right)$ & 0,029 & 0 & 0,080 & 0 & 0,029 & 0 & 0,029 & 0 \\
\hline $\mathrm{R}^{2}$ & 0,931 & 0,870 & 0,999 & 0,996 & 0,999 & 0,996 & 0,997 & 0,967 \\
\hline
\end{tabular}

(1) $\mathrm{m}$ estimado com relação de dependência de $n$ que possibilita estimativas de $\mathrm{K}(\theta)$. ${ }^{(2)} \mathrm{b}$ equivale a $1 / \beta$ no modelo de Hutson \& Cass (1987). ${ }^{(3)}$ Valores obtidos em laboratório. 
As maiores diferenças observadas nos teores de água ajustados pelos dois modelos foram obtidas na faixa de teor de água mais elevado (Figura 1b). Observa-se que pequenas mudanças no teor de água levam a grande mudança na tensão.

Comparando os valores observados e ajustados de $\theta$ (Figura 2), pode-se observar melhor pelo seu maior ou menor distanciamento da linha 1:1, quanto os ajustes subestimam ou superestimam os dados de teor de água. Tanto no modelo de Hutson \& Cass quanto no modelo de van Genuchten, a regressão com o valor de $\theta$ i torna-se mais favorável por apresentar menor distanciamento em relação à linha 1:1 (melhor coeficiente angular). Análises entre os modelos estudados revelam que o de van Genuchten torna-se mais confiável em comparação ao modelo proposto por Hutson \& Cass, por apresentar coeficiente angular mais próximo de 1 e intercepto mais próximo de zero.

$\mathrm{O}$ valor do índice de confiança c proposto por Camargo \& Sentelhas (1997) para o ajuste de Hutson $\&$ Cass, com $\theta \mathrm{i}$, foi de 0,912 e, sem $\theta \mathrm{i}$, foi de 0,907; para o de van Genuchten, com $\theta$ i, foi de 0,976 e, sem $\theta \mathrm{i}$, foi de 0,970 . Portanto, mesmo assumindo $\theta \mathrm{r}=0$,

(a)

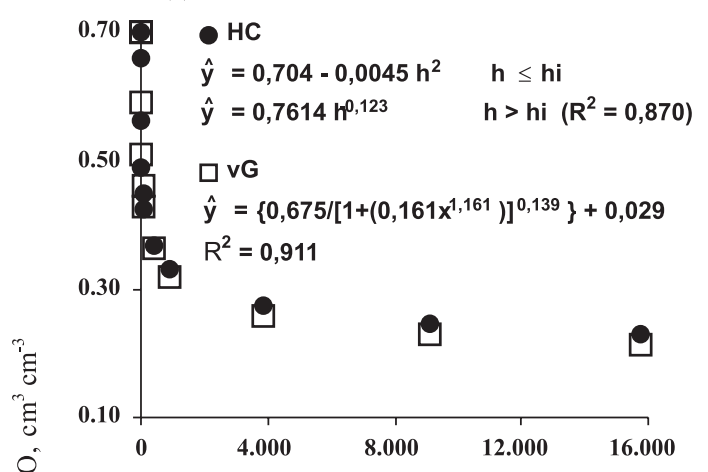

(b)

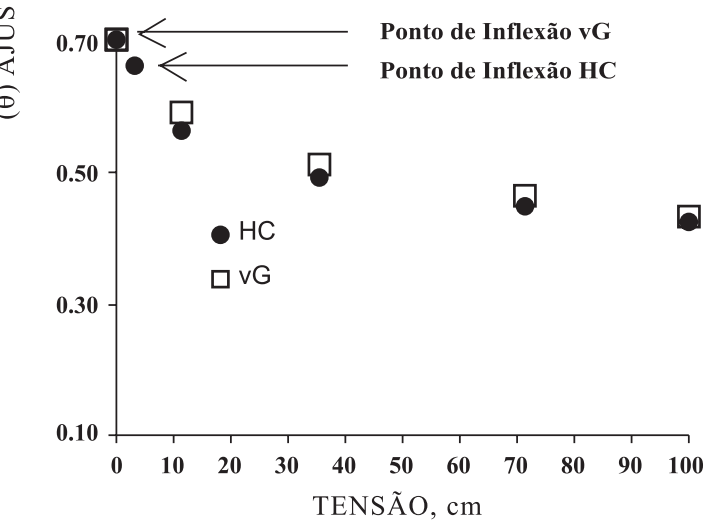

Figura 1. Dados ajustados de teor de água $(\theta)$ e respectivas curvas de retenção de água no solo, obtidos com amostras deformadas, pelo método da centrífuga, considerando as equações propostas por van Genuchten - vG e Hutson \& Cass - HC nos intervalos de tensão de 0 a $16.000 \mathrm{~cm}$ (a) e de 0 a $100 \mathrm{~cm}$ (b). (a)

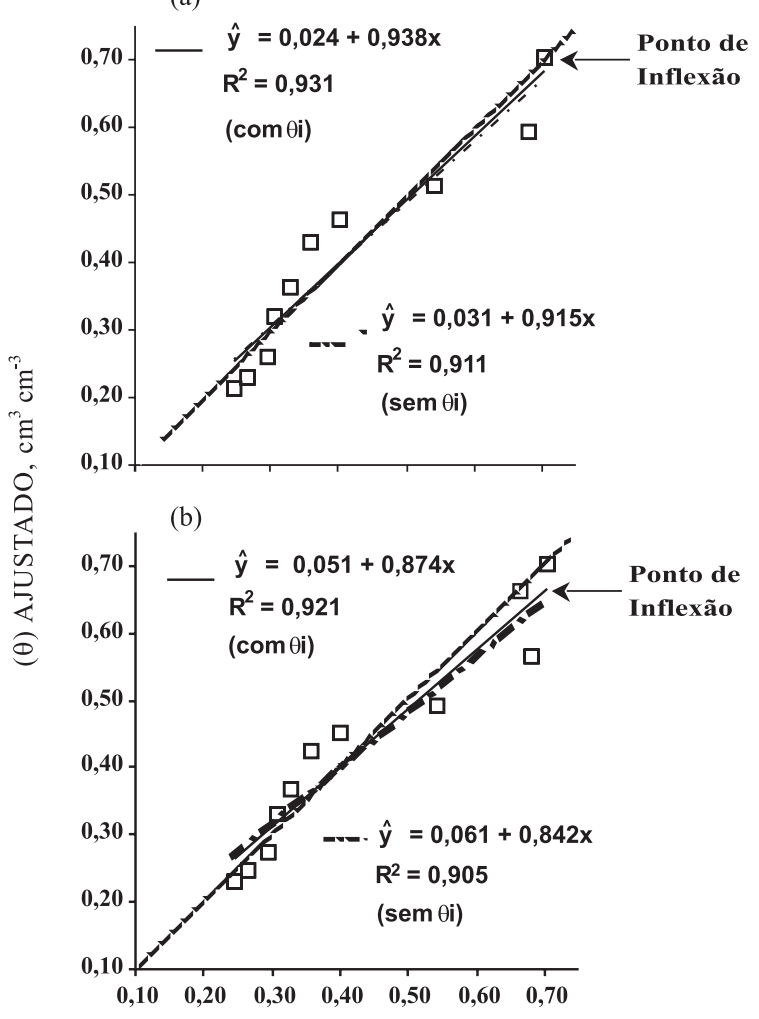

( $\theta)$ OBSERVADO, $\mathrm{cm}^{3} \mathrm{~cm}^{-3}$

Figura 2. Linha 1:1 tracejada, dados observados e ajustados de teor de água $(\theta)$ das curvas de retenção de água no solo pelo método da centrífuga considerando as equações propostas por (a) van Genuchten e (b) Hutson \& Cass.

ambos os modelos apresentaram ótimo desempenho, entretanto com ligeira vantagem para o modelo de van Genuchten. Além disso, a equação de van Genuchten possibilita estimar a relação $K(\theta)$.

Os processos de umedecimento e secagem usando o WP4 diferiram relativamente pouco entre si (Figura 3). Observa-se uma possível existência do fenômeno de histerese, que se confirma com a obtenção do valor do coeficiente angular de 0,939, ou seja, há um certo distanciamento dos dados ajustados em relação à linha 1:1. Tal fato demonstra que, os dados ajustados no processo de umedecimento subestimam os ajustados do processo de secagem. Salienta-se que nos pontos com teor de água mais elevados, os dados ajustados pelo processo de umedecimento são menores do que os de secagem. O fato de os Latossolos apresentarem argilas pouco expansivas e agregados estáveis, mesmo submetidos a processos contínuos de umedecimento e secagem, ajuda a explicar o reduzido efeito de histerese deste solo.

Considerando a água disponível igual à diferença entre o teor de água nas tensões de 15.000 e $100 \mathrm{~cm}$, obteve-se para o processo de secagem um valor de $0,133 \mathrm{~cm}^{3} \mathrm{~cm}^{-3}$, e para o processo de umedecimento $0,118 \mathrm{~cm}^{3} \mathrm{~cm}^{-3}$. Tal fato demonstra que a água disponível 
obtida pelo processo de secagem foi aumentada em $13 \%$ em relação à obtida pelo processo de umedecimento.

Ao analisar o método da centrífuga com amostras deformada e indeformada (Figura 4a,b), observou-se uma acentuada diferença entre as amostras com e sem a sua estrutura original. As diferenças foram acentuadas devido ao baixo coeficiente angular obtido de 0,669. Tal valor demonstra que os dados ajustados de teor de água com amostra indeformada são inferiores ao da deformada, ou seja, amostras com estrutura alterada apresentam teores de água maiores do que aquelas com estrutura inalterada.
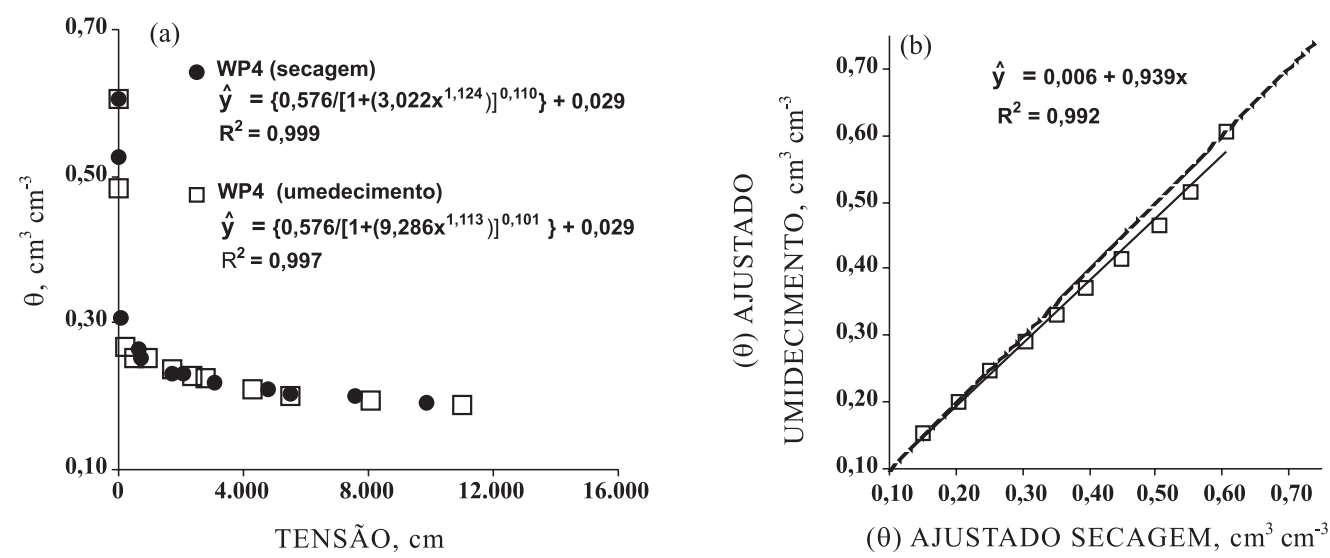

Figura 3. Dados observados de teor de água $(\theta)$ e respectivas curvas ajustadas de retenção de água no solo, pelo modelo de van Genuchten, obtido por um processo de secagem e umedecimento usando amostras deformadas e o método do WP4 (a); linha 1:1 tracejada e dados ajustados no intervalo de $0,15 \leq \theta \leq 0,60 \mathrm{~cm}^{3} \mathrm{~cm}^{-3}$ (b).
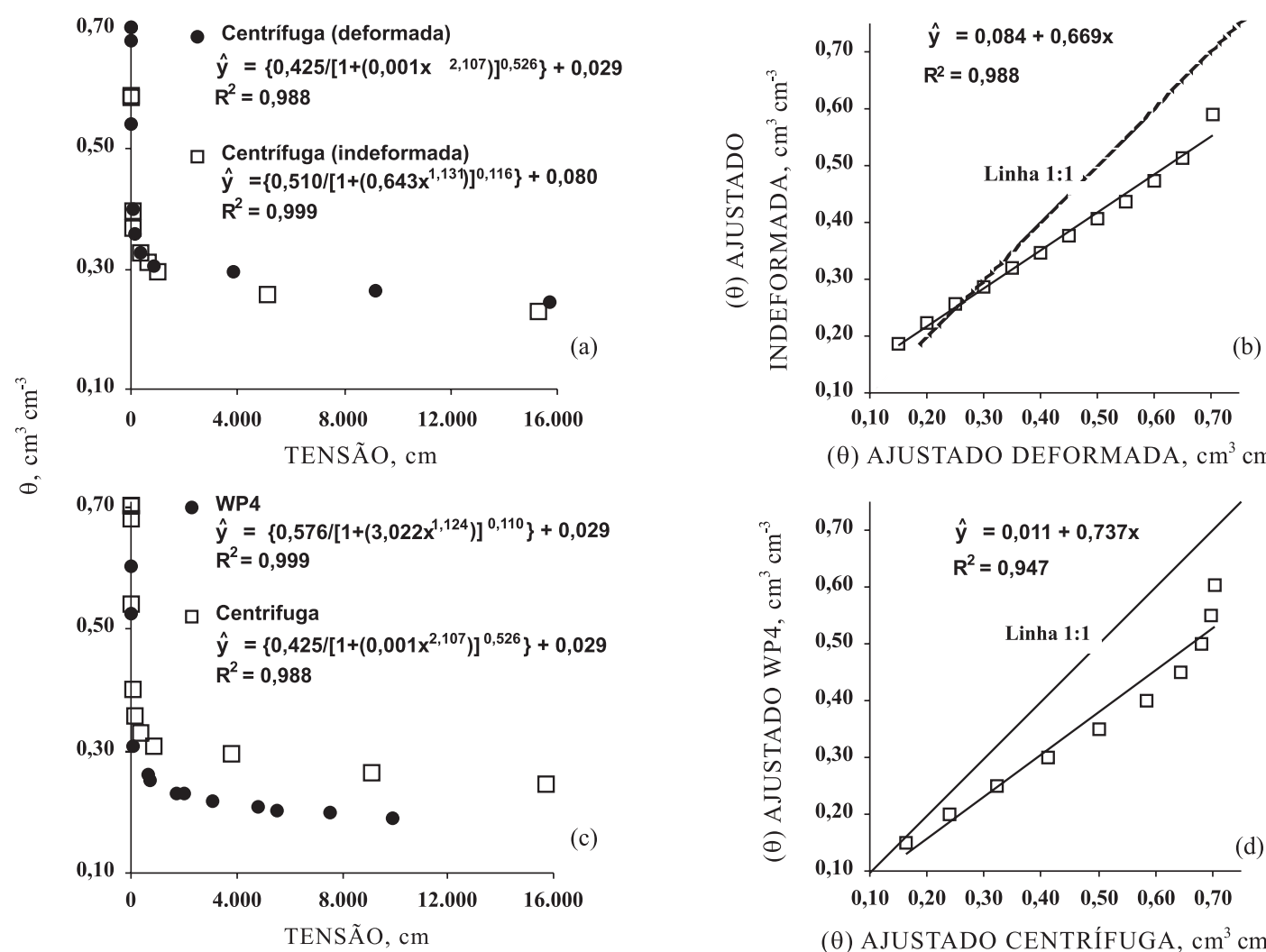

( $\theta$ ) AJUSTADO DEFORMADA, $\mathrm{cm}^{3} \mathrm{~cm}^{-3}$

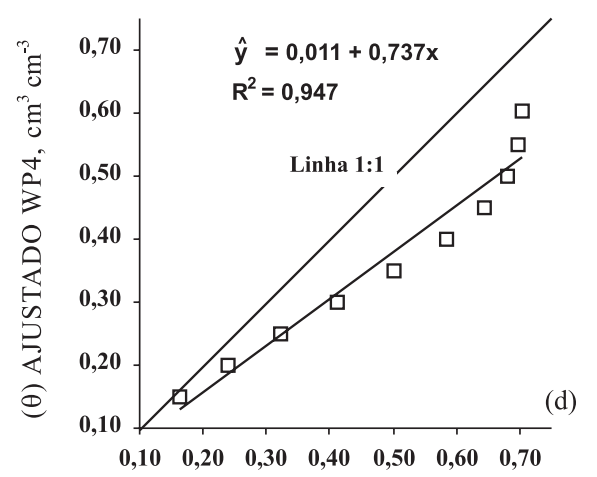

( $\theta$ ) AJUSTADO CENTRÍFUGA, $\mathrm{cm}^{3} \mathrm{~cm}^{-3}$

Figura 4. Dados observados de teor de água ( $\theta)$ e respectivas curvas ajustadas de retenção de água no solo, segundo van Genuchten, em: (a) amostras deformada e indeformada usando o método da centrífuga; (b) respectivos dados de teor de água ajustados no intervalo de $0,15 \leq \theta \leq 0,70 \mathrm{~cm}^{3} \mathrm{~cm}^{-3}$; (c) amostra deformada e processo de secagem, usando os métodos WP4 e centrífuga; e (d) respectivos dados de teor de água ajustados no intervalo de $0,15 \leq \theta \leq 0,70 \mathrm{~cm}^{3} \mathrm{~cm}^{-3}$. 
A água disponível obtida de amostras indeformadas e deformadas, pelo método da centrífuga, foram iguais a 0,141 e $0,228 \mathrm{~cm}^{3} \mathrm{~cm}^{-3}$, respectivamente, proporcionando uma diferença de cerca de $61,7 \%$. Tal diferença pode ser considerável para manejo da irrigação de diversos cultivos. Amostras deformadas podem apresentar, por natureza, maior microporosidade e teores de água do solo, em tensões de $100 \mathrm{~cm}$. Tal fato ajuda a explicar este resultado.

Ao comparar os métodos do WP4 e da centrífuga, com teor de água disponível de 0,133 e $0,228 \mathrm{~cm}^{3} \mathrm{~cm}^{-3}$, respectivamente, observam-se nítidas diferenças entre eles, constatadas tanto visualmente, entre os dados observados de um e outro método (Figura 4c), quanto pelo coeficiente angular obtido de 0,737 (Figura 4d). Houve um distanciamento considerável dos dados ajustados para ambos os métodos em relação à linha 1:1, principalmente em teores de água mais elevados. Assim, pode-se afirmar que os dados obtidos pelo método WP4 subestimam aqueles obtidos pelo método da centrífuga, que, por sua vez, também deformam a amostra em função da rotação aplicada pelo equipamento. Ambos os métodos apresentam a possibilidade de erros sistemáticos e aleatórios. Todavia mais comparações entre esses dois métodos em vários tipos de solos precisam ser realizadas para avaliar as possibilidades de substituição de um método pelo outro com maior eficiência.

\section{CONCLUSÕES}

1. Os modelos de van Genuchten e Hutson \& Cass tiveram ótimo desempenho, com base na determinação do parâmetro "c" proposto por Camargo \& Sentelhas (1997), mas o de van Genuchten se apresentou ligeiramente melhor nas situações estudadas.

2. As curvas de retenção elaboradas por processos de secagem e umedecimento, usando o método WP4, proporcionaram maior teor de água disponível no processo de secagem e uma reduzida presença do fenômeno da histerese.

3. As curvas de retenção elaboradas com amostras deformadas e indeformadas usando o método da centrífuga proporcionaram maior teor de água disponível ao utilizar amostra deformada.

4. As curvas de retenção elaboradas pelos métodos do WP4 e centrífuga proporcionaram teores de água disponível menores para o método WP4.

\section{LITERATURA CITADA}

ADÁMOLI, J.; MACEDO, J.; AZEVEDO, L.G. \& NETTO, J.M. Caracterização da região dos Cerrados. In: GOEDERT, W.J. Solos dos cerrados. Tecnologias e estratégias de manejo. Brasília, Centro de Pesquisa Agropecuária dos Cerrados, 1985. p.33-74.
BIOPHYSICAL RESEARCH INSTRUMENTATION. Disponível em: <http://www.decagon.com/wp4/ wptheory.html >. Acesso em 21 de Setembro de 2004.

BROOKS, R.H. \& COREY, A.T. Properties of porous media affecting fluid flow. J. Irrig. Drainage Division Am. Soc. Civil Eng., 92:61-88, 1966.

CAMARGO, A.P. \& SENTElhas, P.C. Avaliação do desempenho de diferentes métodos de estimativa da evapotranspiração potencial no Estado de São Paulo. R. Bras. Agrometeorol., 5:89-97, 1997.

CAMPBELL, G.S. A simple method for determining unsaturated conductivity from moisture retention data. Soil Sci., 117:311-314, 1974.

CARSEL, R.F. \& PARRISH, R.S. Developing joint probability distribution of soil water retention characteristics. Water Res. Res., 24:755-769, 1988.

DOURADO NETO, D.; JONG VAN LIER, Q.; BOTREL, T.A. \& LIBARDI, P.L. Programa para confecção da curva de retenção de água no solo utilizando o modelo de Genuchten. Eng. Rural, 1:92-102, 1990.

DOURADO NETO, D.; NIELSEN, D.R.; HOPMANS, J.W.; REICHARDT, K.; BACCHI, O.O.S. \& LOPES, P.P. Soil water retention curve. SWRC, version 3.00. Piracicaba, 2001.

EMPRESA BRASILEIRA DE PESQUISA AGROPECUÁRIA EMBRAPA. Centro Nacional de Pesquisa de Solos. Manual de métodos de análise de solos. 2.ed. Rio de Janeiro, 1997. 212p.

EMPRESA BRASILEIRA DE PESQUISA AGROPECUÁRIA EMBRAPA. Sistema brasileiro de classificação de solos. Brasília, Embrapa Produção de Informação; Embrapa Solos, 1999. 412p.

FREITAS JÚNIOR, E. \& SILVA, E.M. Uso da centrífuga para a determinação da curva de retenção de água do solo, em uma única operação. Pesq. Agropec. Bras., 19:1423-1428, 1984.

HUTSON, J.L. \& CASS, A. A retentivity function for use in soil-water simulation models. J. Soil Sci., 38:105-113, 1987.

IWATA, S.; TABUCHI, T. \& WARKENTIM, B.P. Soil water interactions. Mechanisms and applications. New York, Marcell Decher, 1988. 380p.

KLUTE, A. Water retention: Laboratory methods. In: BLACK, C.A., ed. Methods of soil analysis. I. Physical and mineralogical methods. Madison, American Society of Agronomy, Soil Science Society of America, 1986.p.635662.

LEIJ, F.I.; RUSSEL, W.B. \& LESCH, S.M. Close-form expression for water retention and conductivity data. Ground Water, 35:848-858, 1997.

MORAES, S.O.; LIBARDI, P.L. \& DOURADO NETO, D. Problemas metodológicos na obtenção da curva de retenção. Sci. Agríc., 50:383-392, 1993. 
MUALEM, Y. A new model for predicting the hydraulic conductivity of unsaturated porous media. Water Res. Res., 12:513-522, 1976.

RICHARDS, L.A. \& FIREMAN, M. Pressure plate apparatus for measuring moisture sorption and transmission by soils. Soil Sci., 56:395-404, 1943.

SANTANA, M.S. Crescimento inicial de duas cultivares de cafeeiro adensado influenciado por níveis de irrigação localizada. Brasília, Universidade de Brasília, 2003. 50p. (Tese de Mestrado)
SANTOS, M.N. Influência de diferentes sistemas de manejo nos teores de carbono orgânico e de nutrientes e no tamanho e distribuição de poros em um Latossolo Vermelho-escuro argiloso na região dos cerrados. Brasília, Universidade de Brasília, 1997. 133p. (Tese de Mestrado)

SILVA, E.M. A test of the independent domain theory for predicting moisture hysteretic paths. Davis, University of California, 1980. 165p. (Tese de Mestrado)

van GENUCHTEN, M.T. A Closed-form equation for predicting the hydraulic conductivity of unsaturated soils. Soil Sci. Soc. Am. J., 44:892-898, 1980. 